\title{
Pentaclorofenol: toxicología y riesgos para el ambiente
}

\author{
Irasema Castillo Morales ${ }^{1}$ \\ Cristina Bárcenas Pazos²
}

\begin{abstract}
RESUMEN
El pentaclorofenol (PCP) y su sal sódica, el pentaclorofenato de sodio (NaPCP), son hidrocarburos halogenados de amplio espectro de toxicidad, muy persistentes, usados para la preservación de la madera contra hongos, bacterias e insectos. EI PCP y el NaPCP son sustancias de alta toxicidad aguda para humanos que aumenta por la presencia de impurezas como las clorodibenzodioxinas y los clorodibenzofuranos. EI PCP tiene graves efectos a largo plazo: es mutágeno, causa alteraciones en los sistemas inmunológico y endocrino, aumenta la susceptibilidad a infecciones y altera las funciones reproductivas. Existen numerosos estudios sobre los efectos adversos de estas sustancias en la salud humana y de los ecosistemas causados por su toxicidad, persistencia, capacidad de bioacumulación y por la toxicidad de sus impurezas. Estos estudios son la base técnica para que el PCP y sus derivados estén severamente restringidos 0 totalmente prohibidos en numerosos países. En México, el PCP está autorizado para usos forestal, urbano e industrial como preservador de madera; su venta debe estar restringida y su aplicación y manejo deben hacerse bajo la responsabilidad de un técnico autorizado. Existe mucha desinformación entre la población acerca de los riesgos que
\end{abstract}

representa para la salud y el ambiente, el manejo inadecuado de estas sustancias y de sus residuos, por lo que este trabajo pretende difundir la información técnica que existe sobre este tema y recomendar la aplicación de las normas y regulaciones nacionales e internacionales aplicables al manejo de esta sustancia.

PALABRAS CLAVE:

Pentaclorofenol, toxicología, ecotoxicología.

\section{ABSTRACT}

Pentachlorophenol (PCP) and its salt, sodium pentachlorophenate (NaPCP) are very persistent halogenated hydrocarbons with a wide spectrum of toxicity and which are used primarily to protect timber from fungi, bacteria and insects. PCP and $\mathrm{NaPCP}$ are chemicals with a high acute toxicity to human beings increased substantially by impurities such as chlorodibezodioxins and chlorodibenzofurans. PCP has long term adverse effects, is mutagenic, causes alterations in immunological and endocrine systems; it increases susceptibility to infections and reproductive dysfunction. There are several studies on the adverse effects of these substances on human and ecosystems health caused by its

2 Departamento de Sistemática Vegetal. Instituto de Ecología, A.C.. Apdo. Postal 63. Xalapa, Ver., 91000 México.

Manuscrito recibido para su publicación el 3 de Septiembre de 1997. 
persistence, bioaccumulation potential and the toxicity of the impurities. These works are the technical basis of the severe restrictions or even total ban status of PCP and its derivatives and residues in several countries. In Mexico, PCP is authorized for forest, urban and industrial applications as a wood preserver. PCP must be purchased and used only by or with the surveillance of a certified technician. There is a significant lack of information among people about the environmental and health risks from the misuse and inadequate disposal of PCP, NaPCP and its residues. The aim of this work is to diffuse the available technical information on this subject and recommend the application of the national and international legislative regulations on PCP use.

KEY WORDS:

Pentachlorophenol, toxicology, ecotoxicology

\section{INTRODUCCION}

El pentaclorofenol (PCP) y su sal sódica, el pentaclorofenato de sodio (NaPCP), son hidrocarburos clorados con un amplio espectro de toxicidad que se han utilizado como herbicidas, insecticidas, alguicidas, bactericidas, molusquicida, defoliantes, preservadores de madera y como agente antimicrobiano en diversos productos (Wall y Stratton, 1994; RIPQPT, 1988).

Por sus múltiples usos el PCP y el $\mathrm{NaPCP}$ se han empleado en grandes volúmenes y hay evidencias de que esto ha causado efectos adversos en la salud de los seres humanos, en los animales y en los ecosistemas.

\section{OBJETIVOS}

En este trabajo se describen el uso, la toxicidad y los efectos del PCP y se señalan las características que presenta el uso de esta sustancia en el país con los siguientes objetivos:

a) Difundir la información disponible sobre los riesgos del uso del Pentaclorofenol con el fin de que los usuarios adopten las medidas preventivas establecidas en la legislación.

b) Señalar la falta de información técnica y comercial accesible sobre esta sustancia en México

c) Señalar la falta de estudios en el país sobre la presencia de este sustancia en el ambiente y sus repercusiones epidemiológicas.

\section{ANTECEDENTES}

El pentaclorofenol es un plaguicida que se ha usado en gran parte del mundo en aplicaciones industriales, agrícolas y domésticas. La compañía Monsanto utilizó el PCP para la protección de la madera desde 1935; a partir de 1936 se fabricó a escala industrial y en 1948 obtuvo el registro en la Agencia de Protección Ambiental de Estados Unidos (EPA), como preservador de madera. (Wilkinson, 1979; USEPA, 1988). Las características físicas y químicas más importantes del PCP, su sinonimia y su proceso de producción se presentan en el Apéndice.

Uso del PCP: En 1978 el PCP y el NaPCP ocuparon el segundo lugar en la lista de plaguicidas usados en mayor volumen en los Estados Unidos y se estimó que su uso generó un ahorro de 7,500 millones de dólares al alargar la vida útil de la madera (Ranga-Rao, 1978); sin embargo, desde 1989, la empresa Vulcan Chemicals, ubicada en Wichita, Kansas, es la única empresa que produce PCP para surtir a las más de cien plantas de preservación de madera de Estados Unidos que aún utilizan este preservador (Van Strum y Merrell, 1989; PANNA, 1989). 
En la Tabla 1 se muestra el volumen utilizado de PCP para preservar madera en diferentes países, estas cantidades son indicativas de su eficacia para prevenir el deterioro de la madera aunque, por lo que puede observarse, su uso presenta una considerable disminución a nivel mundial entre 1977 y 1986.

En México no se produce PCP, pero sí se formula el producto concentrado procedente de Estados Unidos en maquiladoras ubicadas en ciudades fronterizas en las que han ocurrido derrames e intoxicaciones personales en varias ocasiones (PANNA, 1989). EI PCP formulado en estas plantas regresa a Estados Unidos de donde se distribuye a México, diversos países de Asia y de América Central y del Sur (PANNA, 1995). Aunque el PCP se ha utilizado en México desde 1960, no hay disponible para la población en general ningún registro, actual o pasado, oficial o de las empresas que lo importan, acerca de la cantidad que ha ingresado al país, el uso que se le dado o la forma en que se ha distribuido en el país.

EI PCP se aplica a la madera mediante dos tipos de métodos: el método con presión que consiste en introducir la madera en tanques herméticamente cerrados (autoclaves) a los que se les aplica primero vacío y luego la solución preservadora a presión. La madera impregnada de PCP con este proceso es adecuada para condiciones de alto riesgo de deterioro, ya que el PCP penetra en la madera y puede sellarse para evitar la volatilización. Actualmente, no hay en México plantas de preservación de madera que utilicen este proceso.

Tabla 1. Cantidad de pentaclorofenol utilizado en diferentes países

\begin{tabular}{||c|c|c|c||}
\hline AÑO & LUGAR & CANTIDAD $(\mathrm{Kg})$ & REFERENCIA \\
\hline 1975 & Estados Unidos & $79,200,000$ & 1 \\
\hline 1977 & Estados Unidos & $22,680,000,000$ & 2 \\
\hline 1978 & Canadá & 4,000 a 5,000 & 2 \\
\hline 1978 & Estados Unidos & 19800,000 & 1 \\
\hline 1979 & Estados Unidos & $18,021,000$ & 1 \\
\hline 1979 & Japón & 301,000 & 3 \\
\hline 1980 & Japón & $11,352,000$ & 3 \\
\hline 1980 & Africa & 250,000 a 300,000 & 1 \\
\hline 1980 & Brasil & 502,000 & 4 \\
\hline 1981 & Brasil & 400,000 & 4 \\
\hline 1983 & Estados Unidos & 24,300 & 6 \\
\hline 1985 & Estados Unidos & $11,390,000$ & 2 \\
\hline 1986 & Estados Unidos & 23000000 & 5 \\
\hline 1986 & Europa & 8000 & 5 \\
\hline 1986 & Otros países & $35000-40000$ & 5 \\
\hline 1995 & Estados Unidos & 32205000 & 7 \\
\hline \hline
\end{tabular}

Fuentes:

1. Nicholas y Cockroft, 1982

5. Fiedler et al., 1991

2. Ranga-Rao, 1978

6. ATSDR, 1989

3. Amemiya y Cockroft, 1982

7. NCAMP, 1996

4. Cavalcante y Cockroft, 1984 
En los métodos sin presión la aplicación del PCP es por aspersión, brocha o inmersión con lo que se obtiene madera adecuada para condiciones de bajo riesgo de deterioro.

En México, de manera generalizada, la aplicación del PCP se hace con métodos sin presión, por lo que no penetra profundamente en la madera y puede volatilizarse constantemente hacia el ambiente.

Impurezas: Durante la producción industrial del PCP se generan compuestos como el hexaclorobenceno (HCB), las policlorodibenzodioxinas (PCDD) y los policlorodibenzofuranos (PCDF) que son contaminantes del PCP concentrado y que también se encuentran en las presentaciones comerciales. Estos subproductos son sumamente tóxicos y poseen un alto riesgo ambiental (Alwicker, 1991).

Generación y eliminación de desechos: Los desechos contaminados con PCP se generan durante la producción industrial, el uso en la industria maderera y a partir de los sobrantes de la madera tratada con procesos sin presión a nivel artesanal y doméstico. En México, las fuentes de ingreso de residuos de PCP al ambiente son el manejo inadecuado de los desechos, la incineración de los residuos de PCP y de la madera tratada con esta sustancia que provoca, además de su volatilización a la atmósfera, la formación de PCDD y PCDF. El ingreso de estos residuos al ambiente es difuso por provenir de numerosas fuentes en cantidades pequeñas, lo que hace imposible su tratamiento y es el origen de la exposición de la población general.

En las fuentes industriales de desechos de PCP como las formuladoras, la aplicación de un tratamiento a las aguas

de desecho puede reducir significativamente la exposición de la población y el ingreso de este compuesto al ambiente. Hay numerosos trabajos sobre metodología para el tratamiento de efluentes contaminados con PCP que puede ser adaptada tecnológicamente a diversas condiciones (Lamar et al., 1990; Higson y Focht, 1990; Jacobsen et al, 1991; Mueller et al., 1991; Middaugh et al., 1991; Balfanz y Rehm, 1991; Wise, 1986).

Rutas potenciales de ingreso al ambiente: El ingreso del PCP al ambiente puede llevarse a cabo por diversas rutas; en la figura 1 se presenta un esquema general del origen, el movimiento y la distribución del pentaclorofenol, sus residuos e impurezas en el ambiente.

La EPA (1989) estimó que, de 1989 a 1993, ingresaron al ambiente alrededor de 52 toneladas de PCP provenientes principalmente de la industria de explosivos (43\%), preservación de madera (20\%) y el resto, de otras industrias (37\%). Se han hecho estimaciones acerca de la distribución de los residuos de PCP en el ambiente y se calcula que se está distribuido de la siguiente manera: $48 \%$ en el suelo, $45 \%$ en sedimentos, $5.5 \%$ en el agua y $1.4 \%$ en el aire (NCAMP, 1996; Hattemer-Frey y Travis, 1989).

EI PCP posee características que le proporcionan una gran capacidad para contaminar el manto freático: baja solubilidad (20 mg/l) y mayor densidad que la del agua $\left(1.98 \mathrm{~g} / \mathrm{cm}^{3}\right)$. Estas características le permiten pasar a la subsuperficie como una fase no acuosa (Rouse, 1990); asimismo, su presión de vapor permite que se volatilice a partir de la madera tratada y su coeficiente de partición agua/octanol que es de 5.01 (Davies y Dobbs, 1984) indica que esta sustancia puede acumularse en organismos $y$ sustratos ambientales.

Exposición de la población humana: Los trabajadores que manejan PCP en la industria de tratamiento de madera, los aserraderos y otros trabajadores que 


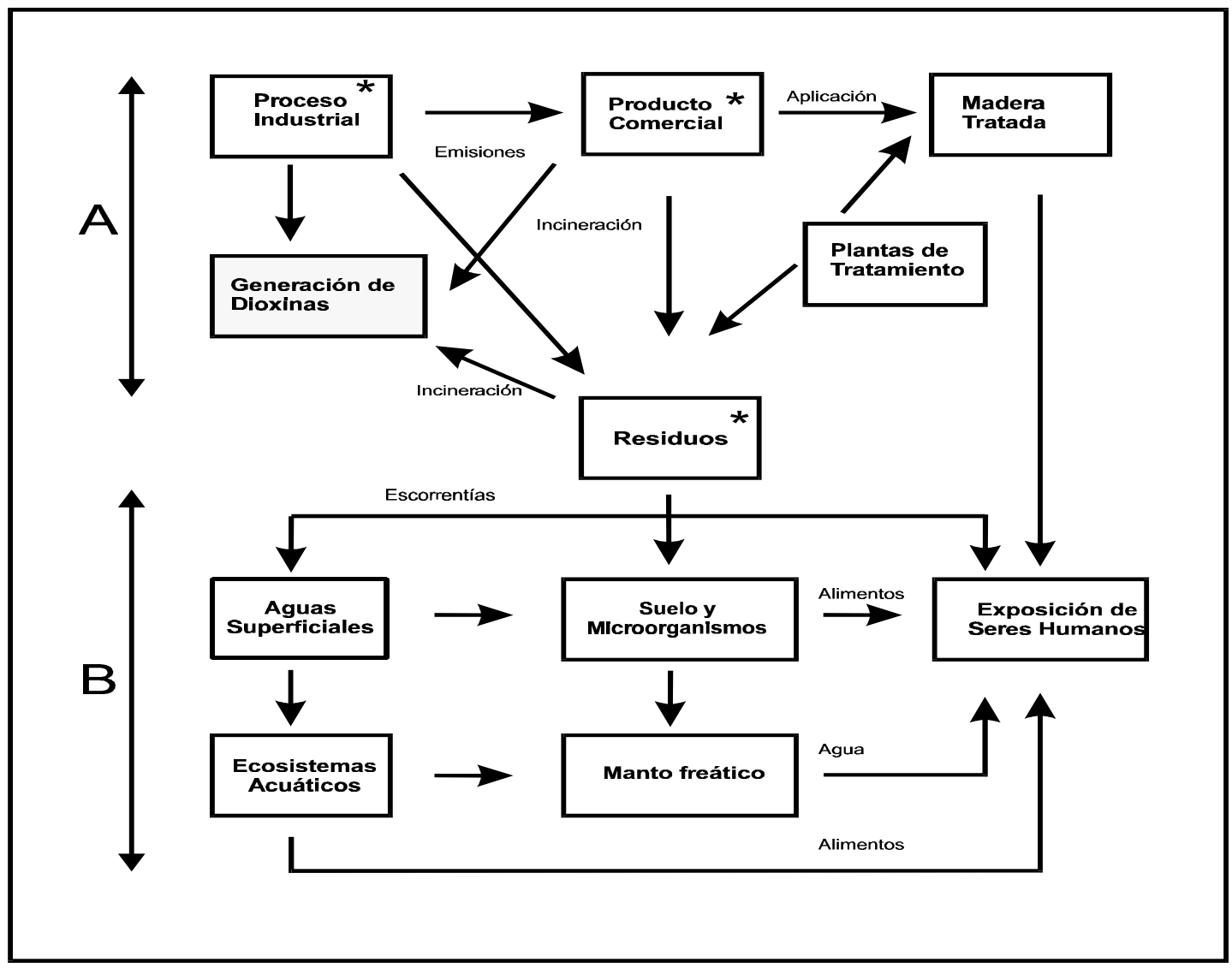

Figura 1. Origen, movimiento y distribución del PCP y sus residuos en el ambiente. A. Origen, B. Distribución de los residuos, * Presencia de Dioxinas.

utilizan PCP o madera tratada son los que pueden estar más expuestos a esta sustancia, principalmente a través de la exposición dérmica y respiratoria, ya que la madera tratada libera PCP que se moviliza del centro de la madera hacia la superficie de donde se evapora, dejando sus impurezas y productos de degradación (Butler y Frank, 1991; USEPA, 1990; Williams, 1982; Kauppinen y Lindroos, 1985; Jones, 1986). La Agencia para Registro de Sustancias Tóxicas y Enfermedades ha estimado que la exposición de los trabajadores es de entre 0.9-14 mg/día (ATSDR, 1989).
Aunque la mayoría de la población no está expuesta directamente al PCP, puede haber exposiciones a bajas concentraciones de este compuesto en el ambiente de trabajo, en casas construidas con madera tratada $\left(0.5-104 \mathrm{mg} / \mathrm{m}^{3}\right)$, por el aire, dentro y fuera de las casas; a través del agua potable $(0.02 \mathrm{mg} /$ día) y de los alimentos (0.1-6 mg/día) y por el contacto directo con madera tratada (ASTDR, 1989). Se han encontrado residuos de PCP en el $79 \%$ de las muestras de orina de grupos de población sin exposición específica (Murphy et al., 1983); estos residuos provienen de los alimentos y el agua, ya que el PCP es un contaminante frecuente en granos, frutas, papas, leche y otros productos 
animales (USEPA, 1988). En estudios realizados por Frank et al. (1990), para determinar residuos de plaguicidas en grasas animales se encontró que el PCP es una de las sustancias detectadas con mayor frecuencia en las muestras de alimentos (35\%).

Se han presentado casos extremos de exposición al PCP, PCDD y PCDF debido a derrames accidentales $y$ al manejo irresponsable de los desechos industriales que han afectado a poblaciones asentadas cerca de sitios en los que se han depositado desechos industriales contaminados con PCP y dioxinas (Kimbrough, 1984; Gough, 1991; Raiger y Bois, 1993).

\section{Toxicología}

El pentaclorofenol es un compuesto lipofílico, persistente y acumulable y está considerado como uno de los contaminantes orgánicos prioritarios desde 1977 (USEPA, 1979). El PCP causa un desacoplamiento de la fosforilación oxidativa que es un proceso básico de la respiración; su toxicidad no es específica por lo que se le considera biocida.

En el ser humano, la exposición al PCP puede ser por las vías oral, dérmica y respiratoria; una vez absorbido, se distribuye en todo el organismo y tiende a acumularse en el hígado, riñones, cerebro y grasa (Braun et al., 1977; Grimm et al., 1981). En la Tabla 2 se presenta la vida media del pentaclorofenol en algunos mamíferos.

La exposición aguda al PCP provoca irritaciones locales, efectos sistémicos, reacciones alérgicas, alteraciones inmunológicas y gastrointestinales y en caso de intoxicaciones fatales se han visto daños severos en órganos como hígado y riñones; los síntomas de intoxicación aguda son: aumento de la temperatura corporal, sudoración abundante, dolor abdominal y náuseas, daños en el sistema nervioso central y, en casos extremos, paro cardíaco (ASTDR, 1989).

\section{Toxicidad aguda}

El PCP está clasificado por la Organización Mundial de la Salud como un compuesto que, en estado puro, posee alta toxicidad (Clasificación lb). En la Tabla 3 se presenta la Dosis Letal Media (DLM) de la sustancia grado técnico (con una pureza mayor al $90 \%$ ) para mamíferos. Los datos para rata corresponden a los valores mínimo y máximo obtenido en ensayos con animales de experimentación en diferentes condiciones fisiológicas como edad y peso, entre otras. La toxicidad aguda, crónica y a largo plazo del PCP se incrementa significativamente con las impurezas que contienen las presentaciones comerciales.

\section{Toxicidad a largo plazo}

EI PCP puede causar intoxicación crónica en trabajadores de aserraderos y en las personas que viven en habitaciones hechas de madera tratada (USEPA, 1988). EI PCP se ha relacionado con anemia aplástica y leucemia, es neurotóxico, afecta adversamente las funciones reproductivas y el desarrollo fetal (USEPA, 1988; Roberts, 1990) y se ha encontrado evidencia de que el PCP puede bioacumularse en el organismo humano (Dow Chemical Co., 1969).

Por otra parte, varios grupos de trabajadores dedicados al tratamiento de madera, agricultores y aplicadores de plaguicidas presentaron un aumento significativo en la incidencia de infecciones (Kleinman et al., 1986; Mcchonnachie y Zahalsky, 1991) y deficiencia renal causada por la exposición crónica a PCP (Begley et al., 1977).

Se han observado efectos adversos por la exposición a PCP a mediano y largo plazo, los más importantes se resumen a continuación: aberraciones cromosómicas en trabajadores (Schrag y Dixon, 1985; USEPA, 1988); defectos congénitos en animales de experimentación (USEPA, 1988; Williams, 1982) y varios estudios han demostrado que el PCP, el tetraclorofenol y la tetraclorohidroxiquinoleina, que es el 
Tabla 2. Vida media del pentaclorofenol

\begin{tabular}{|c|c|}
\hline ESPECIE & VIDA MEDIA (horas) \\
\hline Peces & 10 \\
\hline Mono & 78 \\
\hline Humano & $30-50$ \\
\hline
\end{tabular}

Vida Media. Periodo que requiere un organismo para eliminar el $50 \%$ de una sustancia.

Fuente: Mercier, 1981.

Tabla 3. Toxicidad aguda del pentaclorofenol y del pentaclorofenato de sodio

\begin{tabular}{||c|c|c|c|c|c||}
\hline \hline \multicolumn{7}{|c||}{ DOSIS LETAL MEDIA DE PENTACLOROFENOL $\left(\mathrm{DL}_{50}{ }^{*}\right)$} \\
\hline Especie & Oral & Dérmica & Respiratoria & $\mathrm{IP}^{* \star}$ & Subcutánea \\
\hline Rata & $25-200(1)$ & $96-320(1)$ & $10-225(1)$ & $56(2)$ & $100(2)$ \\
\hline Ratón & $130(1)$ & $261(1)$ & $355(1)$ & & \\
\hline Conejo & $130(1)$ & & & & \\
\hline \multicolumn{7}{|c|}{ DOSIS LETAL MEDIA DE PENTACLOROFENATO DE SODIO $\left(\mathrm{DL}_{50}\right)^{*}$} \\
\hline Rata & $210(2)$ & $164(2)$ & $72(2)$ & \\
\hline
\end{tabular}

* $\quad \mathrm{DL}_{50}$ : Dosis Letal Media: Dosis capaz de matar al $50 \%$ de una población expuesta $(\mathrm{mg} / \mathrm{kg})$

** IP: Intraperitoneal

Fuente: 1. Wagner, 1983; 2. Sax, 1979.

principal producto de degradación del PCP, pueden dañar el material genético. (Williams, 1982; USEPA, 1988a).

Los estudios realizados para determinar la carcinogenicidad del PCP no han sido realizados de manera adecuada, por lo que el PCP está clasificado por la EPA y por la Agencia Internacional de Investigación sobre el Cáncer (IARC), como un probable carcinógeno para humanos que requiere más estudios, (USEPA, 1990); sin embargo, el hexaclorobenceno (HCB) y la HxCDD, impurezas del PCP, son carcinógenos reconocidos y existen evidencias epidemiológicas de que la exposición simultánea a clorofenoles y dioxinas o plaguicidas contaminados con estas sustancias incrementan el riesgo de sarcoma de tejido suave y de linfoma (Kerkvliet et al. , 1982).
Toxicidad de las dioxinas (PCDD) y dibenzofuranos (PCDF)

Las intoxicaciones masivas de seres humanos y aves de corral y los accidentes industriales relacionados con estas sustancia, han hecho evidente que la presencia de PCDD y PCDF en los productos comerciales de PCP es un riesgo potencial muy alto, ya que estas sustancias, además de ser extremadamente tóxicas de manera aguda poseen graves efectos adversos a mediano y largo plazo.

La exposición de seres humanos a dosis altas de dioxinas produce daño en el hígado, neuropatía periférica, profiria cutánea tardía y alteraciones sicológicas. (Ayres et al., 1985.); también pueden causar pigmentación e incremento en el peso y el volumen del hígado y los riñones (Johansen et al., 1973; Gough, 1991; Kimbrough, 1984), en algunos casos, las PCDD y los 
PCDF causan cloracné que es una enfermedad cutánea persistente, así como alteraciones en la capacidad reproductiva. En primates la exposición a TCDD causa pérdida de peso, alopecía, edema, lesiones hepáticas y fetotoxicidad. (Ayres et al. 1985). La exposición de la población humana ocurre principalmente a través de los alimentos, especialmente los lácteos y cárnicos, ya que las PCDD y los PCDF son lipofílicos y tienen potencial de biocumulación (Jiménez et al., 1991). La concentración límite autorizada de dioxinas en presentaciones comerciales de PCP en Estados Unidos es de 4 partes por millón (ATSDR, 1989); en México, a pesar de estar autorizado el uso de PCP no hay regulaciones con respecto al contenido de dioxinas.

\section{Efectos en el ambiente}

El uso inapropiado, los derrames accidentales y la disposición inadecuada de los residuos del PCP han causado la contaminación de numerosos sustratos ambientales. En los lugares en donde se ha usado el PCP en procesos industriales, es frecuente encontrar residuos de este compuesto en concentraciones altas al igual que de sus impurezas (PCDD y PCDF) en aire, agua y suelos. Todos estos compuestos son resistentes a la degradación biológica lo que los hace muy persistentes en el ambiente y permite que sus residuos sean arrastrados por el agua de lluvia, se adhieran a las partículas del suelo o incluso ingresen directamente en los cuerpos de agua.

Los residuos de $\mathrm{PCP}$ son prácticamente ubícuos en el ambiente, sin embargo, la importancia de sus efectos es mayor en los siguientes compartimentos ambientales:

Suelo: EI PCP es muy persistente y tiene una vida media en el suelo superior a los 5 años y se calcula que en este sustrato se encuentra alrededor del $48 \%$ del PCP que se ha liberado en el ambiente; sus residuos se unen fuertemente a las partículas del suelo y esto favorece que migre a suelos neutros y alcalinos (USEPA, 1988). Por otro lado, en campos de cultivo inundados, la presencia de residuos de PCP eliminó hasta el $90 \%$ de algas de los géneros de algas Charay Nitelle (Butler, 1977). EI PCP puede movilizarse de la capa superior del suelo hacia el aire por evaporación y puede lixiviarse y contaminar el manto freático. El PCP se degrada en el suelo si está expuesto a la luz solar y las bacterias y la velocidad de degradacion aumenta en condiciones anaeróbias, temperatura alta y presencia de materia orgánica. Las concentraciones de PCP de 20 ppm pueden inhibir la actividad de los microorganismos del suelo y continuar inhibiéndola hasta 48 dias después (Zelles et al, 1985). EI PCP es muy tóxico para las plantas y puede ser absorbido por ellas a partir del suelo y causar la pérdida del delicado equilibrio que existe entre las comunidades biológicas que habitan el suelo (Engst et al., 1977).

Ecosistemas acuáticos: Se han detectado residuos de PCP en agua y sedimentos de ecosistemas acuáticos en los que se calcula que se encuentra alrededor del $45 \%$ del PCP que ha ingresado al ambiente, también se han encontrado en agua de escorrentía e incluso en sistemas de agua potable así como en organismos acuáticos (Seidler et al., 1986). Debido a que el PCP tiende a unirse a los sedimentos y a la materia particulada, puede moverse del suelo al agua superficial y a la profunda. EI PCP puede persistir en el agua durante lapsos de varias horas a días y su degradación se lleva a cabo básicamente en la superficie del agua. Si el PCP ingresa a los cuerpos acuáticos en concentraciones elevadas pueden provocar mortandad de peces, invertebrados y vegetales $y$ se ha observado que concentraciones de 2 a 10 microgramos/litri de PCP en agua pueden inhibir el crecimiento de algunos peces (Niimi y Cho, 1983).

Peces: EI PCP es muy tóxico para peces de agua fría y de clima templado y se ha observado que los valores bajos de $\mathrm{pH}$ y las 
temperaturas elevadas aumentan su toxicidad en exposiciones crónicas (Fisher, 1991). En la Tabla 4 se presentan los valores de la Concentración Letal Media de PCP y NaPCP para algunos organismos acuáticos.

Las concentraciones de PCP detectadas hasta ahora en ríos, corrientes y agua superficial (Tabla 5) están por debajo de las concentraciones que se consideran tóxicas, sin embargo, se han encontrado concentraciones entre 130 y 970 microgramos/l de PCP en los efluentes y en la corriente receptora de plantas de tratamiento de madera en USA (Wylie et al., 1990); estas concentraciones están por encima de la $\mathrm{CL}_{50}$ para varias especies, por lo que pueden afectar gravemente a las poblaciones animales y vegetales.

Varios estudios sobre PCP y sus productos de degradación han mostrado que el PCP se acumula rápidamente en organismos y en sedimento a partir de la columna de agua y persiste por varios meses; también se ha observado que el pH estuario puede favorecer la incorporación de PCP presente en el sedimento a través de su adsorción en el detritus y la asimilación en las algas bénticas y el zooplancton. Estos trabajos han ayudado para concluir que el PCP tiene un importante potencial de bioacumulación (Brown, 1978; Davies y Dobbs, 1984; Niimi y Cho, 1983).

Tabla 4. Concentración letal media de pentaclorofenol y pentaclorofenato de sodio para organismos acuáticos

\begin{tabular}{||l|c|c||}
\hline \multicolumn{1}{|c|}{ ESPECIE } & $\begin{array}{c}\mathrm{PCP} \\
\mathrm{CL}_{50}^{*}(\mathrm{mg} / \mathrm{l})\end{array}$ & $\begin{array}{c}\mathrm{NaPCP} \\
\mathrm{CL}_{50}(\mathrm{mg} / \mathrm{l})\end{array}$ \\
\hline Ceriodaphnia reticulata($(A)$ & & $0.22-0.55(1)$ \\
\hline Daphnia magna $(A)$ & & $0.47-1.12(1)$ \\
\hline Daphnia pulex $(A)$ & & $1.04-1.14(1)$ \\
\hline Palemonetes pugio $(C)$ & $0.515,96 \mathrm{~h}(2)$ & $0.196,96 \mathrm{~h}(2)$ \\
\hline Pimephales promelas $(P)$ & $0.19,96 \mathrm{~h}(2)$ & $0.21,48 \mathrm{~h}(2)$ \\
\hline Oncorhynchus mykiss $(P)$ & $0.23,96 \mathrm{~h}(2)$ & $0.048-0.1,96 \mathrm{~h}(2)$ \\
\hline
\end{tabular}

$\mathrm{CL}_{50}$. Concentración Letal Media: Concentración en el agua capaz de matar al $50 \%$ de una población experimentalmente expuesta durante un lapso definido (mg/l),

A. Artrópodo; C. Crustáceo; P. Pez

Fuentes: 1. Hall et al., 1986; 2. Ramammoorthy y Baddaloo, 1995.

Tabla 5. Concentraciones de PCP detectadas en el ambiente

\begin{tabular}{||l|c||}
\hline \multicolumn{1}{|c|}{ SUSTRATO } & CONCENTRACION \\
\hline Ríos y corrientes & $0.01-16 \mathrm{ppb}$ \\
\hline Aguas superficiales & $1.3-12 \mathrm{ppb}$ \\
\hline Aguas marinas & $0.02-11 \mathrm{ppt}$ \\
\hline Manto freatico & $0.06 \mathrm{ppt}-0.64 \mathrm{ppb}$ \\
\hline Aire & $0.93-28 \mathrm{ppt}$ \\
\hline
\end{tabular}

ppb: nanogramos/kg; ppt: picogramos $/ \mathrm{kg}$

Fuente: Howard, 1991. 


\section{Regulaciones para el uso del Pentaclorofenol}

Internacionales: Desde 1988, el PCP está incluido dentro del grupo de plaguicidas considerados de alto riesgo por el conjunto de sus graves y diversos efectos en la biota; sin embargo, en nuestro país existe muy poca información acerca del manejo del producto y de los residuos generados a partir de su uso. El PCP está totalmente prohibido en un total de 27 países, entre ellos Alemania, Suecia y Dinamarca, y severamente restringido en otros 14 , entre ellos México (PANNA, 1994; PANNA, 1995); sin embargo, la efectividad de estas restricciones y prohibiciones es muy variable ya que en algunos países las regulaciones son cumplidas estrictamente mientras que en otros, el uso ilegal de plaguicidas prohibidos es muy común.

En Estados Unidos, la Agencia de Protección Ambiental tiene al PCP en un proceso de revisión para eliminar sus usos mas riesgosos (PANNA, 1991) y la Ley de Control de la Contaminación considera al PCP, sus isómeros, hidratos, mezclas, soluciones y desechos de madera tratada, como sustancias y residuos peligrosos que deben cumplir con las regulaciones y los permisos necesarios para su manejo, transporte, almacenamiento y disposición (IRPTC, 1993).

\section{Nacionales}

Producto comercial: El Catálogo Oficial de Plaguicidas (CICOPLAFEST, 1994) que contiene todos los plaguicidas registrados cuya importación, distribución y uso están autorizados en México, tiene clasificado al PCP como un plaguicida de uso restringido que sólo puede ser adquirido en las empresas comercializadoras mediante la presentación de una recomendación escrita de un técnico oficial o privado que haya sido autorizado por el gobierno federal. El manejo y la aplicación de esta sustancia se efectuarán bajo la responsabilidad y supervisión del técnico autorizado que lo haya recomendado (CICOPLAFEST, 1994).

EI PCP está autorizado para uso forestal, urbano e industrial por lo que puede ser aplicado en bosques y madera, ciudades y zonas habitacionales (sin incluir el uso doméstico) y en el procesamiento de productos y subproductos así como para el cuidado de áreas industriales. Todas las presentaciones comerciales de PCP tienen una Categoría Toxicológica de III, es decir, son sustancias muy tóxicas que tienen una Dosis Letal Media de entre 20 y $200 \mathrm{mg} / \mathrm{kg}$ de peso, por lo que deben cumplir con las normas nacionales de etiquetado.

Residuos de Pentaclorofenol: Con respecto a los residuos generados por el uso del pentaclorofenol la Norma Oficial Mexicana NOM-CRP-001-ECOL/93 (SEDESOL, 1993) considera como residuos peligrosos del giro industrial de preservación de la madera a los lodos sedimentados del tratamiento de aguas en procesos que utilizan clorofenol y pentaclorofenol, asi como los residuos del proceso de cloración en la producción de preservadores para madera. En la lista de residuos peligrosos generados por fuentes no específicas están incluidos los residuos de tri, tetra, o pentaclorofenol provenientes de su producción o de su uso como reactante, producto intermedio o componente de una formulación. Esta norma también establece las concentraciones de estas sustancias que les confieren las características de peligrosidad a los residuos y efluentes.

Una vez que se establece que los residuos de un proceso son tóxicos debe observarse el cumplimiento del Reglamento en materia de Residuos Peligrosos de la Ley General del Equilibrio Ecológico y la Protección al Ambiente (SEDESOL, 1988) que establece que la SEMARNAP es la autoridad encargada de vigilar el cumplimiento de las disposiciones adecuadas para el manejo de residuos 
peligrosos, en este caso, los generados por el uso de PCP y NaPCP, autorizar a los generadores de residuos peligrosos y establecer y mantener actualizado un sistema de información sobre la generación de residuos peligrosos.

Este reglamento determina que quien pretenda realizar obras o actividades que generen o en las que se manejen residuos peligrosos deben contar con una autorización de la Secretaría, inscribirse en un registro de generadores, llevar una bitácora, envasar, etiquetar y almacenar adecuadamente sus residuos peligrosos y darles una disposición final adecuada en un confinamiento de residuos peligrosos.

Es una obligación del generador dar aviso a las autoridades correspondientes cuando ocurra un derrame, haya infiltraciones, descarga o vertimiento de residuos peligrosos durante cualquiera de sus operaciones y es una atribución de la Secretaría el derecho de realizar actos de inspección en las empresas para verificar el cabal cumplimiento de las disposiciones legales.

Algunos autores que han realizado estudios sobre los efectos del PCP concluyen que, por las características toxicológicas y ecotoxicológicas, es inaceptable el riesgo para el ambiente y la salud humana, ocasionado por el uso de productos que contienen PCP, PCDD y PCDF (Jorens y Schepens, 1993). Sin embargo, las autoridades nacionales encargadas de autorizar el uso de plaguicidas han considerado conveniente el uso del PCP, siempre y cuando se lleve a cabo en condiciones adecuadas para la prevención de riesgos (CICOPLAFEST, 1994).

En México no hay información oficial sobre la cantidad PCP utilizado a nivel industrial o doméstico y las empresas que lo importan no proporcionan datos al respecto. Esto impide dar una perspectiva cuantitativa de la importancia del PCP como factor de riesgo ambiental y de salud. Sin embargo, a manera de dato indicativo, cuando el PCP ha estado entre las sustancias químicas presentes en accidentes industriales en México, se han reportado volúmenes de hasta 1,500 litros en una sola planta formuladora (PANNA, 1993).

\section{CONCLUSIONES}

El volumen de PCP potencialmente utilizado en México es mucho menor que el que puede ser utilizado en países con una industria maderera formal importante; sin embargo, existen circunstancias, como las que a continuación se mencionan, que pueden elevar considerablemente el riesgo durante el uso de esta sustancia:

1) Existe desconocimiento entre la población en general de los riesgos que existen durante el uso, almacenamiento y la aplicación de PCP; esto es de suma importancia ya que la mayor parte de este producto se vende a granel y se aplica a nivel artesanal o doméstico en procesos sin presión, lo que genera exposición de los comerciantes, de los aplicadores y de las personas que permanecen expuestas a la madera tratada que se utiliza en casas habitación 0 en instalaciones agropecuarias.

2) Faltan técnicos capacitados en la aplicación adecuada del PCP en madera en procesos sin presión que tengan, además, capacitación en la prevención de riesgos.

3) Falta difusión y capacitación para la aplicación de las regulaciones en el manejo de PCP, sus residuos y de los residuos de la madera tratada.

4) Falta aplicación del control en la venta del producto comercial y, en general, no se cumplen las normas sobre etiquetado y publicidad comercial del producto como puede apreciarse en diversas etiquetas $y$ folletos que carecen de la información toxicológica básica como las emitidas por 
Pentaclorofenol, S.A. de C.V. en 1985, 1986 y 1993.

5) Como resultado de todo lo anterior, existe un manejo inadecuado de los desechos contaminados con PCP; no se ponen en práctica las medidas de seguridad adecuadas y por consiguiente el vertimiento de residuos se lleva a cabo en el drenaje y suelos, se depositan al aire libre y se incineran, con la consecuente liberación de residuos al ambiente.

6) Otra circunstancia que agrava el riesgo potencial del uso de PCP en México y que hace necesaria su evaluación, es la falta de un sistema de vigilancia epidemiológica que registre enfermedades y patologías relacionadas con la exposición ocupacional, accidental o doméstica al PCP o a otras sustancias tóxicas.

\section{RECOMENDACIONES}

Para disminuir los riesgos generados por el uso del PCP para la salud humana y el ambiente se hacen las siguientes recomendaciones

A) Promover la difusión de los riesgos potenciales a la salud y el ambiente causados por el uso inadecuado del PCP, el NaPCP y otras sustancias tóxicas.

B) Promover la difusión de las medidas preventivas generales para el uso de sustancia tóxicas (Apéndice).

C) Promover la capacitación de Técnicos $y$ encargados de comercios y almacenes que manejen productos que contengan PCP, NaPCP y otras sustancias tóxicas.

D) Promover la difusión y aplicación de la normatividad y las regulaciones establecidas en la aplicación del PCP, por el Catálogo Oficial de Plaguicidas en lo que se refiere al control de su uso y de su comercialización, por la Norma técnica NOM-CRP-001ECOL/93 que considera al PCP y NaPCP, sus subproductos y desechos como residuos peligrosos y por la normatividad nacional e internacional sobre el etiquetado de sustancias tóxicas.

E) Promover la investigación y desarrollo de procesos de preservación de madera que sean alternativos del uso de sustancias de alto riesgo ambiental.

\section{REFERENCIAS}

Alwicker, E.R. 1991. Some laboratory experimental designs for obtaining dynamic property data on dioxins. Sci. Total Environ. 104. p:76-72.

Amemiya, S. y R. Cockcroft. 1982. Wood preservation in Japan. Styrelsen for Teknisk utveckling information nr 272. Stockholm, Sweden p:27-28.

ASTDR. 1989. Agency for Toxic Substances and Disease Registry. Pentachlorophenol. Public Health Statement.

Ayres, S. M., K. B Webb, R. G. Evans y B. Mikes. 1985. Is 2,3,7,8-TCDD (dioxin) a carcinogen for humans?. Environ. Health Perspect. 62:329-335.

Balfanz, J. y H. J. Rehm. 1991. Biodegradation of 4-chlorophenol by adsorptive inmobilized Alcaligenes sp D-7-2 in soil. Appl Microbiol. Biotechnol. 35:662-668.

Begley J., E. L. Reichert, M. N. Rashad y H. W. Klemmer. 1977. Association between renal function tests and pentachlorophenol exposure. Clin. Toxicol. 11:97-106.

Braun, W.H., J. D. Young, G. E. Blau y P. J. Ghering. 1977. The pharmacokinetics 
and metabolism of pentachlorophenol in rats. Toxicol. Appl. Pharmacol. 41:395.

Brown, A.W.A. 1978. Ecology of pesticides. John Wiley. $525 \mathrm{p}$.

Butler, G.L. 1977. Algae and Pesticides. Res. Rev. 66:15-56.

Butler, K.M. y R. Frank. 1991. Pentachlorophenol residues in porcine tissues following preslaughter exposure to treated wood shavings. J. Food. Protec. 54(6):448-450.

Cavalcante, M. y R. Cockcroft. 1984. Wood preservation in Brazil. Swedish National Board for Technical Development. Information No. 445. Stockholm Sweeden. p:15.

CICOPLAFEST. 1994. (Comisión Interinstitucional para el control del Proceso y Uso de Plaguicidas, Fertilizantes y Sustancias Tóxicas). Catálogo Oficial de Plaguicidas CICOPLAFEST. SARH, SEDESOL, SRIA. SALUD, SECOFIN, México. D.F. $481 \mathrm{p}$.

Davies, R.P. y A. J. Dobbs. 1984. The prediction of bioaccumulation in fish. Water. Res. 18(10):1253-1262.

Dow Chemical Company. 1969. Antimicrobial agents. Dowcide 2 Antimicrobial. Dow Chemical Co. Midland MI.

Engst, R., P. M. Macholz y M. Kuyawa. 1977. Recent state of lindane metabolism. Res. Rev 68:59-90.

Fiedler, H., O. Hutzinger y C. W. Timms. 1990. Dioxins: sources of environmental load and human exposure. Toxicol. Environ. Chem. 29:157-234.
Fisher, S.W. 1991. Changes in toxicity of threee pesticides as a function of environmental $\mathrm{pH}$ and temperature. Bull. Environ. Contam. Toxicol. 46: 1977-202.

Frank, R. et al. 1987. Monitoring wood shaving litter and animal products for polychlorophenol residues, Ontario, Canada, 1978-1986. Draft Report. Ontario, Canada: Ministry of Agriculture and Food.

Gough, M. 1991. Human Health effects: what the data indicate. Sci. Total Environ. 104:129-158.

Grimm, H. G., B. Schellman, K.H. Schaller y K. Gossieer. 1981 . Pentachlorophenol concentrations in tissues and body fluids of normal persons. Zentralbl Bakteriol. Mikrobiol. Hyg. 174 (1-2): 77-90.

Hall, W. S. , R.L. Paulson, L. W. Hall y D.T. Burton. 1986. Acute toxicity of $\mathrm{Cd}$ and NaPCP to daphnids and fish. Bull. Environ. Contam. Toxicol 37(2):308316.

Hattemer-Frey, H.A. y C.C. Travis. 1989. Pentachlorophenol: environmental partitioning and human exposure. Arch. Environ. Contam. Toxicol. 18:482-489.

Higson, F. K. y D. D. Focht. 1990. Bacterial degradation of ring-chlorinated acetophenones. Appl. Environ. Microbiol. 56(12): 3678-3685.

Howard, P.H. 1991. Handbook of Environmental Fate and Exposure Data for Organic Chemicals. Volume III. Lewis Publishers. Chelsea, MI. 350 p.

IRPTC. 1993. (International Register of Potentially Toxic Chemicals). Legal Files. UNEP. Ginebra, Suiza. 
Jacobsen, B.N. et al. 1991. Microbial degradation of pentachlorphenol and lindane in laboratory scale activated sludge reactors. Wat. Sci. Technol. 23:349-356.

Jiménez, B., M.J. González y L.M. Hernández. 1991. Presence of PCDDs and PCDFs in food for human consumption: A review. Food Chem. 39:257-271.

Johansen, P.H., R.A. S. Mathers, J. A. Brown y P. W. Colgan. 1985. Mortality of early stages of largemouth bass, Micropterus salmoides due to pentachlorophenol exposure. Bull. Environ. Contam. Toxicol. 34:377-384.

Jones, R.D. 1986. Absorption study of pentachlorophenol in persons working with wood preservatives. Hum. Toxicol. 5:189-194.

Jorens, P.G. y P.J.C. Schepens. 1993. Human pentachlorophenol poisoning. Hum. Exp. Toxicol. 12:479-495.

Kauppinen, T. y L. Lindroos. 1985. Chlorophenol exposure in sawmills. Am. Ind. Hyg. Assn. J. 46(1):34-38.

Kerkvliet, N.I. et al. 1982. Immunotoxicity of pentachlorophenol (PCP): increased susceptibility to tumor growth in adult mice fed technical PCP-contaminated diets. Toxicol. Appl. Pharmacol. 62:5564.

Kimbrough, R.D. 1984. The epidemiology and toxicology of TCDD. Bull. Environ. Contam. Toxicol. 33:636-647.

Kleinman, et al. 1986. Industrial hygiene, chemical and biological assessment of exposure to chlorinated phenolic sap stain control agent. Am. Ind. Hyg. Assn. 42(12): 731-741.

Lamar, R.T., M. J. Larsen y K. K. Kirk. 1990. Sensitivity to and degradation of pentachlorophenol by Phanerochaete spp. Appl. Environ. Microbiol. 56(11):3519-3526.

Mcchonnachie P.R. y A.C. Zahalsky. 1991. Immunological consequences of exposure to pentachlorophenol and creosote contaminated groundwater by physical extraction. Chemical and biological assessment. Arch. Environ. Health 46(4):249-253.

Mercier, M. 1981. Organochlorine Pesticides. P. 170. Pergamon Press. $285 \mathrm{p}$.

Middaugh, D.P.; J.G. Mueller y R.L. Thomas. 1991. Detoxification of PCP and creosote contaminated groundwater by physical extraction: chemical and biological assess. Arch. Environ. Contam. Toxicol. 21:233-244.

Mueller, J.G.; D.P. Middaugh, S.E. Lantz y P.J. Chapman. 1991. Biodegradation of creosote and PCP in contaminated groundwater: chemical and biological asessment. Appl. Envrion. Microbiol. 57(5):1277-1285.

Murphy, R.S. et al. 1983. Selected pesticide residues or metabolites in blood and urine specimens from a general population survey. Env. Health. Perspect. 48:81-86.

NCAMP. 1996. National Coalition against the Misuse of Pesticides. Chemicals at a Glance. Penta. A Report About their Toxic Trail and Safer Alternative.

Nicholas, D.D. y R. Cockcroft. 1982. Wood preservation in the USA. Styrelsen for Teknisk utveckling information nr 288. Stockhlom, Sweden. p: 24-26.

Niimi, A.J. y C.Y. Cho. 1983. Laboratory and field analysis of pentachlorophenol (PCP) accumulation by salmonids. Water Res. 17(12)1791-1795. 
PANNA. 1989. Pesticide Action Network, Pentachlorophenol spills at Mexican maquiladora. PAN North America, American Regional Center. San Francisco, CA.

PANNA. 1990. Pesticide Action Network, Dirty dozen pesticides fact sheets. PAN North American Regional Center. San Francisco, CA.

PANNA. 1991. Pesticide Action Network, EPA drafts "safer" pesticide policy. PANNA Outlook. PAN North America Regional Center. San Francisco, CA.

PANNA. 1993. Pesticide Action Network, Mexican victims file suit in U.S. Court. Updates Service. PAN North America Regional Center. San Francisco, CA.

PANNA. 1994. Pesticide Action Network, European Union ban on PCP considered. Updates Service. PAN North America Regional Center. San Francisco, CA.

PANNA. 1995. Pesticide Action Network, 1995 Demise of the dirty dozen. PAN North America Regional Center. San Francisco, CA.

Pentaclorofenol, S.A. de C. V. 1985. Vida Madera S.R. Boletín VIDSR 4/85. México, D.F.

Pentaclorofenol, S.A. de C.V. 1986. PentaMaderol. Boletín PEN-9786. México, D.F.

Pentaclorofenol, S.A. de C.V. 1993. Más vida a sus maderas con Vida MaderaLPU. Boletín V-LPV 10793. México, D.F.

Rammamorthy, S. y E. Baddaloo. 1995. Handbook of chemical tocixity profiles of biological species. CRC Press. $450 p$.
Raiger, B. G. y F. Y. Bois. 1993. Pentachlorophenol carcinogenicity: extrapolation of risks from mice to humans. Human Exp. Toxicol. 12:215225.

Ranga-Rao, K., 1978. Pentachlorophenol: Chemistry, Pharmacology and Environmental Toxicology. Plenum Press. $402 \mathrm{p}$.

RIPQPT. 1988. Registro Internacional de Productos Químicos Potencialmente Tóxicos. Pentaclorofenol y Pentaclorofenato de sodio. Boletín Vol. 9 No. 1. Programa de las Naciones Unidas para el Medio Ambiente (PNUMA) RIPQPT. Suiza. p: 38-41.

Roberts, H.J. 1990. Pentachlorophenolassociated aplastic anemia, red cell aplasia, leukemia and other blood disorders. J. Florida Med. Assn. 77(2):86-90.

Rouse, J.V. 1990. Dense non-aqueous liquid (DNAL). Behavior and its implications on remedial options. Control. Proceed. 86th Ann. Meeting Am. Wood Preserv. Assoc.

Sax, N.I. 1979. Dangerous properties of Industrial Materials. Van Nostrand Pub. 850 p.

Schrag, S.D. y R.L. Dixon. 1985. Occupational exposures associated with male reproductive dysfunctions. Ann. Rev. Pharmacol. Toxicol. 25:567592.

SEDESOL. 1988. Secretaría de Desarrollo Social. Ley General del Equilibrio Ecológico y la Protección al Ambiente. Reglamento en Materia de Residuos Peligrosos. Edit. Delma. Cap. V: 131152.

SEDESOL. 1993. Secretaría de Desarrollo Social. Norma Oficial Mexicana NOMCRP-001-ECOL/93. Que establece las 
caraccterísticas de los residuos peligrosos, el listado de los mismos y los límites que hacen a un residuo peligroso por su toxicidad al ambiente. Diario Oficial de la Federación. México. $30 \mathrm{p}$.

Seidler, J. J., M. Landau, F. E. Dierberg y R. H. Pierce. 1986. Persistence of pentachlorophenol in a wastewaterestuarine aquaculture system. Bull. Environ. Contam. Toxicol. 36(1):101108.

Stecher, D.G., M. Widholz, D.S. Leahy, D.M. Bolton, L.G. Eaton. 1968. The Merck Index; Merck Co. Inc. Rahway, NJ, USA p. 792.

USEPA. 1979. Environmental Protection Agency. Suspended, Cancelled, and Restricted Pesticides. Washington, D.C. Office of Pesticides and Toxic Substances.

USEPA. 1990. Environmental Protection Agency. Identification and listing of hazardous waste. Wood Preserving. Federal Register 55(235):5045050490.

USEPA. 1988. Health Advisory Office. Pentachlorophenol. Rev. Environ. Contam. Toxicol. 104:183-194

USEPA.1988a. Office of Drinking Water. 2,3,7,8-Tetrachlorodibenzo-p-dioxina. Rev. Environ. Contam. Toxicol. 107:147-163.
Van Strum, C. y P. Merrell. 1989. The politics of penta. Greenpeace. Seattle, WA.

Wall, A.J. y G. W. Stratton. 1994. Arsenic effects on the bacterial degradation of pentachlophenol. Chemosphere 28(3):559-566.

Wagner, S. L. 1983. Clinical Toxicology of Agricultural Chemicals, Noyes Data Corporation.

Williams, P. L. 1982. Pentachlorophenol, an assessment of the occupational hazard. Am. Ind. Hyg. Assn. J. 43:799810.

Wilkinson, J. G. 1979. Indutrial Timber Preservation. Associated Business Press. Londres, Inglaterra. p: 136, 298-300 y 303.

Wise, E.D. 1986. RCRA Surface Impoundment Options for Creosote and Pentachlorophenol wood treating facilities. American Wood-Preserver's Association. Philadelphia, PA. 7 p.

Wylie, G.D., S.E. Finger y R.W. Crawford. 1990. Toxicity of municipal wastewater effluents contaminated by pentachlorophenol in southwest Missouri. Environ. Pollut. 64:43-53.

Zelles, L. et al. 1985. Side effects of some pesticides on non-target soil microorganisms. Environ. Sci. Health B20(5):457-488. 


\section{APENDICE}

Propiedades Físicas y Químicas del Pentaclorofenol y Pentaclorofenato de Sodio

El pentaclorofenol puro se presenta como cristales en forma de ahujas, polvo, escamas y gránulos que van de color blanco a tostado claro, gris o pardusco, es una sustancia volátil, no corrosiva a los metales y se descompone ante la presencia de agentes oxidantes fuertes y la luz solar. Tiene un peso molecular de 266.5, un punto de fusión entre 187 y 191 /C, su punto de ebullición está entre 309 y 310 /C y una presión de vapor de 0.00017 atm a $20 / C$ y de $0.14 \mathrm{~atm}$ a $100 / \mathrm{C}$. El pentaclorofenol tiene una densidad de $1.978 \mathrm{~g} / \mathrm{ml}$, es casi insoluble en agua $(0.0018 \%)$ a $27 / C$ y soluble en metanol, etanol, benceno y etilen-glicol; en tanto que el pentaclorofenato de sodio es muy soluble en agua (Stecher et al, 1968, Sax, 1979).

\section{Nombres de Productos Comerciales que Contienen Pentaclorofenol y Pentaclorofenato de Sodio (CICOPLAFEST, 1994; Pentaclorofenol S.A. de C. V., 1985, 1986, 1993).}

$\begin{array}{ll}\text { Ambrotox-0 Concentrado } & \text { Osmose-Triox } \\ \text { Penta-Maderol } & \text { Pentarin L } \\ \text { Pentadragón 50 Pino } & \text { Pentatox } \\ \text { Pentaclorofenol 5 } & \text { Dowcide 7 } \\ \text { Pentalox-S } & \text { Polacida L } \\ \text { Pentamadera } & \text { Mitrol G-ST } \\ \text { Vida Madera LPU } & \text { Polacida } \\ \text { Vida-Madera } & \end{array}$

\section{Método de obtención del Pentaclorofenol}

Proceso Boehringer. La cloración del fenol se lleva a cabo en dos etapas, primero se burbujea cloro a través del fenol a 41 /C con lo que se obtienen tri- $y$ tetraclorofenoles, posteriormente se aumenta la temperatura a 54 /C para mantener líquida la reacción mientras se aumenta la cloración. Mediante este proceso se obtiene entre 88 y $96 \%$ de pentaclorofenol y de 4 a $12 \%$ de tetraclorofenoles que bajo ciertas condiciones se dimerizan y generan subproductos como las dibenzo-p-dioxinas (PCDD) y los dibenzofuranos clorados (PCDF) (Rouse, 1990, Dow Chemical, 1969).

\section{Medidas para disminuir los efectos adversos del PCP y el NaPCP en la salud humana y el ambiente}

CAplicar los plaguicidas siguiendo estrictamente las indicaciones de la etiqueta respecto a dosis, número y técnicas de aplicación y medidas de seguridad (respirador, ropa de protección y guantes).

CNo comer, beber o fumar en las áreas en que se trabaje con plaguicidas, no tocar los ojos, la nariz o la boca mientras se usan plaguicidas y lavar las manos con abundante agua y jabón después de manejar plaguicidas y antes de comer, beber, fumar o usar el servicio sanitario.

CAplicar el plaguicida solamente bajo la supervisión y responsabilidad de un técnico capacitado.

CLimitar la aplicación de plaguicidas al área que es el objetivo del control.

CNo limpiar o lavar los equipos cerca de arroyos y canales, ríos, lagos o cualquier otro cuerpo de agua o directamente en ellos ni arrojar sobrantes o envases vacíos.

CDisponer la eliminación de los envases y los residuos conforme a las medidas propuestas por el Catálogo Oficial de Plaguicidas $y$ el Reglamento sobre Residuos Sólidos de la Ley General del Equilibrio Ecológico y de Protección al Ambiente (CICOPLAFEST, 1994). 
